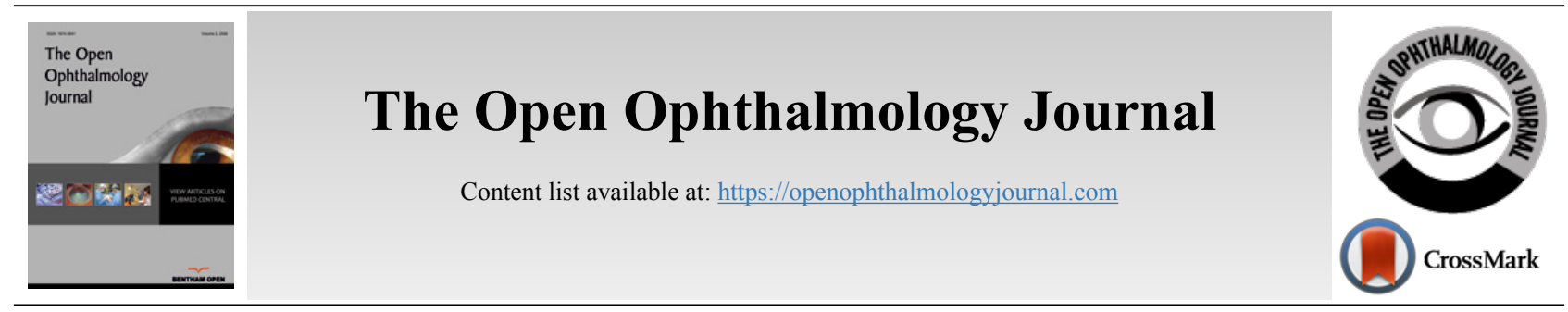

RESEARCH ARTICLE

\title{
Anti-Vascular Endothelial Growth Factor Therapy as an Adjunct to Diabetic Vitrectomy
}

Mushfig Karimov ${ }^{1}$ and Lala Akhundova ${ }^{1, *}$

${ }^{1}$ Department of Diabetic Eye Diseases, Zarifa Aliyeva National Ophthalmology Centre, Baku, Azerbaijan

\begin{abstract}
:
Introduction:

The purpose of this work is to study the efficacy of the preoperative intravitreal administration of bevacizumab as an adjunct to vitrectomy in patients with Proliferative Diabetic Retinopathy (PDR).

Methods:

This retrospective comparative study was performed on 118 eyes (118 patients) with proliferative diabetic retinopathy (PDR), which underwent vitrectomy surgery at the Department of Diabetic Eye Disease at Zarifa Aliyeva National Ophthalmology Centre (Baku, Azerbaijan) in 2015-2019. The main group (the bevacizumab group) included 48 eyes with PDR that received intravitreal administration of bevacizumab (Avastin; Genentech Inc., USA) within one week before vitrectomy; the control group included 70 eyes that did not receive a bevacizumab injection for at least 3 months before the vitrectomy. The minimum follow-up was 12 months.

Results:

In both groups, complete retinal attachment after primary vitrectomy was achieved in all eyes (100\%). Clinically significant intraoperative haemorrhage was observed in the preoperative bevacizumab injection group in $31.2 \%$ and the control group- $51.4 \%, p=0.030$. The preoperative bevacizumab injection reduced the risk of clinically significant haemorrhage by 2.3 times and the need for endodiathermy by 2.7 times $(p=0.031$ and $p=0.024$, respectively). Early vitreous cavity haemorrhage was observed in $15.0 \%$ in the bevacizumab group and in $35.5 \%$ in the control group $(p=0.038)$. The preoperative injection of bevacizumab before vitrectomy reduced the risk of vitreous cavity haemorrhage in the early postoperative period by 3.0 times $(\mathrm{p}=0.036)$.

\section{Conclusion:}

The preoperative use of bevacizumab as an adjunct to diabetic vitrectomy can help reduce the incidence of intraoperative and early postoperative vitreous cavity haemorrhage, which leads to better functional results in the early postoperative period. Over the long-term follow-up period, the effect of the preoperative bevacizumab injections decreases.
\end{abstract}

Keywords: Proliferative diabetic retinopathy, Vitrectomy, Endodiathermy, Vitreous cavity haemorrhage, Anti-VEGF therapy, Intravitreal bevacizumab.

Article History Received: March 31, 2021 Revised: July 09, 2021 Accepted: July 13, 2021

\section{INTRODUCTION}

Diabetic retinopathy is one of the main causes of vision loss among working-age people in developed countries [1]. The disease is characterized by progressive retinal ischaemia, which leads to the development of neovascularization of the retina, optic nerve head and iris, as well as the formation of epiretinal fibrovascular membranes. In the later stages, the con-

\footnotetext{
* Address correspondence to this author at the Department of Diabetic Eye Diseases, Zarifa Aliyeva National Ophthalmology Centre, 32/15 Javadkhan str., Baku, Azerbaijan; Tel: +994503565181; E-mail: akhundova.lale@gmail.com
}

traction of the fibrovascular membranes can cause intraocular haemorrhage and tractional retinal detachment.

Advances in vitreoretinal surgery technologies helped to improve anatomical and functional outcomes after vitrectomy in eyes with severe complications of Proliferative Diabetic Retinopathy (PDR) [2]. Nonetheless, despite the benefits of surgical treatment, the risk of severe intraoperative and postoperative complications, such as recurrent bleeding into the vitreous cavity, remains at a high level [3]. Intraoperative Vitreous Haemorrhage ( $\mathrm{VH})$ deteriorates the visualization of 
the surgical field, impeding complex surgical manoeuvres, prolonging the surgical time and may lead to serious complications, even in the hands of the most experienced surgeons $[4,5]$.

The frequency of recurrent $\mathrm{VH}$ varies from $30 \%$ to $75 \%$, and the cases are usually classified as early ( $\leq 4$ weeks) or late ( $>4$ weeks) recurrent VH [2 - 5]. The source of early VH is the dispersion of the blood from the periphery to the centre of the vitreous cavity from dissected or trimmed fibrovascular membranes during surgery. The source of late $\mathrm{VH}$ is the neovascularization of the sclerotomies, vitreous base, iris, angle and retina. Various techniques have been suggested to reduce the risk of recurrent haemorrhages, including cryotherapy to the sclerotomies, gas or silicone oil endotamponade, with unproven efficacy [6 - 8].

Vascular endothelial growth factor inhibitors (anti-VEGF), such as ranibizumab, bevacizumab, and aflibercept, are commonly used in ophthalmology to treat macular oedema and intraocular neovascularization $[9,10]$. There are many reports in the medical literature on the successful use of anti-VEGF agents to reduce the incidence of intra- and postoperative complications during vitrectomy in patients with PDR. Preoperative intravitreal injection of bevacizumab was reported to reduce intraoperative and postoperative bleeding in PDR cases $[11-16]$. At the same time, some authors consider the effect of preoperative anti-VEGF injections insignificant [17 20].

The aim of this work was to determine the efficacy of the preoperative intravitreal administration of bevacizumab as an adjunct to vitrectomy in reducing the incidence of intraoperative complications and preventing postoperative vitreous cavity haemorrhage in patients with PDR.

\subsection{Plain Language Summary}

\subsubsection{What was known Before}

There are controversial reports in the medical literature on the efficacy of preoperative anti-VEGF injection to reduce the incidence of intra- and postoperative complications in patients with PDR, undergoing vitrectomy surgery.

Bevacizumab, a full-length recombinant humanized monoclonal antibody targeting all VEGF isoforms, is frequently used before vitrectomy to reduce complications without proven efficacy.

\subsubsection{What this Study Adds}

The preoperative use of bevacizumab as an adjunct to diabetic vitrectomy can help to reduce the incidence of intraoperative and early postoperative vitreous cavity haemorrhage, which leads to better functional results in the early postoperative period.

The preoperative injection of bevacizumab reduced the risk of intraoperative haemorrhage by 2.3 times $(\mathrm{p}=0.031)$ and the need for endodiathermy by 2.7 times $(p=0.024)$, but did not influence the duration of surgery.

Intravitreal injections of bevacizumab before surgery also reduced the risk of early postoperative vitreous cavity haemorrhage by 3.0 times $(p=0.036)$, but did not significantly affect the incidence of late vitreous cavity haemorrhage. The incidence of late postoperative vitreous cavity haemorrhage was $16.7 \%$ with preoperative bevacizumab injection and $17.1 \%$ without injection

\section{MATERIALS AND METHODS}

This retrospective comparative study investigated the efficacy of bevacizumab as an adjunct to 23-gauge vitrectomy for complications of PDR. One hundred eighteen Caucasian patients who underwent surgery at the Department of Diabetic Eye Disease at Zarifa Aliyeva National Ophthalmology Centre (Baku, Azerbaijan, Eastern Europe) in 2015-2019 were investigated in this study. The main group (further called the bevacizumab group) included 48 eyes with PDR that received intravitreal administration of bevacizumab (Avastin; Genentech Inc., USA) before vitrectomy; the control group included 70 eyes that did not receive a bevacizumab injection. Inclusion criteria for the main group were: intravitreal injection of anti-VEGF agents within one week before surgery, followup for at least 12 months after primary vitrectomy. The control group included eyes that had not received an injection for at least 3 months before the surgery and were under follow-up for at least 12 months. Exclusion criteria for both groups were: tractional-rhegmatogenous retinal detachment, preoperative visual acuity no higher than light perception, duration of silicone oil tamponade for more than 6 months. If both eyes of the same patient were operated on, the eye with the longer follow-up period was chosen for the study. The demographic data of the patients in the two groups (age, sex, type of diabetes, duration, level of compensation, method of treatment) are shown in Table $\mathbf{1}$.

Table 1. Patient demographics.

\begin{tabular}{|c|c|c|c|c|c|}
\hline \multicolumn{3}{|c|}{ Variables } & $\begin{array}{c}\text { The } \\
\text { Bevacizumab } \\
\text { Group }\end{array}$ & $\begin{array}{c}\text { The } \\
\text { Control } \\
\text { Group }\end{array}$ & $p$-value \\
\hline \multicolumn{3}{|c|}{ Number of Patients } & 48 & 70 & - \\
\hline \multicolumn{3}{|c|}{ Number of eyes } & 48 & 70 & - \\
\hline \multirow[t]{2}{*}{ Sex } & \multicolumn{2}{|l|}{ Male } & $23(47.9 \%)$ & $32(45.7 \%)$ & \multirow{2}{*}{$0.814 *$} \\
\hline & \multicolumn{2}{|c|}{ Female } & $25(52.1 \%)$ & $38(54.3 \%)$ & \\
\hline \multirow[t]{2}{*}{ Age } & \multicolumn{2}{|c|}{ Mean \pm SD } & $54.02 \pm 11.63$ & $57.78 \pm 8.50$ & \multirow[t]{2}{*}{$0.045 \%$} \\
\hline & \multicolumn{2}{|c|}{ Range } & $28-78$ & $25-74$ & \\
\hline \multirow{6}{*}{$\begin{array}{c}\text { Diabetes } \\
\text { mellitus }\end{array}$} & \multirow[t]{2}{*}{ Type } & 1 & $4(8.3 \%)$ & $3(4.3 \%)$ & \multirow[t]{2}{*}{$0.307 \%$} \\
\hline & & 2 & $44(91.7 \%)$ & $67(95.7 \%)$ & \\
\hline & \multicolumn{2}{|c|}{ Duration (Years \pm SD) } & $\begin{array}{c}11.55 \pm 6.54 \\
(0-25)\end{array}$ & $\begin{array}{c}14.12 \pm 6.79 \\
(1-35)\end{array}$ & $0.095 \%$ \\
\hline & \multirow[t]{2}{*}{ Management } & Insulin & $43(89.6 \%)$ & $63(90.0 \%)$ & \multirow[t]{2}{*}{$1.000 \%$} \\
\hline & & Oral & $5(10.4 \%)$ & $7(10.0 \%)$ & \\
\hline & \multicolumn{2}{|c|}{$\begin{array}{c}\text { HbA1c (Glycolysated } \\
\text { haemoglobin, in \%, } \\
\text { range) }\end{array}$} & $\begin{array}{l}8.27 \pm 1.04 \\
(6.3-10.5)\end{array}$ & $\begin{array}{c}7.85 \pm 0.81 \\
(6.0-9.8)\end{array}$ & $0.078 \%$ \\
\hline
\end{tabular}

$<0.05$, the result is highlighted in bold.

\subsection{Intravitreal Injection Technique}

An intravitreal injection of bevacizumab $0.05 \mathrm{ml}(1.25 \mathrm{mg})$ was performed transconjunctivally in the operating room in the 
inferotemporal pars plana using a 27-gauge needle at a distance of 3.5-4 $\mathrm{mm}$ from the limbus in phakic eyes and $3 \mathrm{~mm}$ from the limbus in pseudophakic eyes.

\subsection{Surgical Technique}

Minimally invasive (23-gauge) vitrectomy was performed using the Constellation surgical system (Alcon, USA); BIOM $4 \mathrm{~m}$ (Oculus, Germany) was used to visualize the posterior segment of the eye. In combined cases, cataract phacoemulsification and IOL implantation were performed at the beginning of the surgery. Core vitrectomy was continued with triamcinolone staining of the posterior hyaloid membrane. The anteroposterior tractions were removed first, followed by the tangential tractions. Additional or primary panretinal laser photocoagulation was performed intraoperatively in full in one session. At the end of the surgery, the vitreous cavity was restored with air, a gas-air mixture $\left(20 \% \mathrm{SF}_{6}\right.$ or $\left.14 \% \mathrm{C}_{3} \mathrm{~F}_{8}\right)$, or 1000 centistoke silicone oil. All surgeries were recorded on a camera integrated with the Zeiss Lumera HD microscope for further analysis.

Clinically significant intraoperative haemorrhage was considered as bleeding that impaired visualization of the surgical field, impeding the performance of surgical manipulations in the vitreous cavity and the retina, and when additional manipulations (increasing the intraocular pressure, endodiathermy use, lowering the blood pressure or replacing the intraocular fluid with air) were usually required. Early postoperative haemorrhage was defined as the presence of vitreous cavity bleeding within the first 4 weeks. Late postoperative haemorrhage was defined as the presence of vitreous cavity bleeding that has occurred more than 4 weeks after surgery with a "clear" non-haemorrhage interval.

\subsection{Statistical Analysis}

The data were statistically analysed using SPSS statistical software (version 20.0, IBM, USA). BCVA was defined using the decimal system, and the data were later converted into LogMAR (logarithm of the minimum angle of resolution) units for statistical processing. The quantitative values are presented as the mean \pm standard deviation. To compare mean values across groups, the Mann-Whitney U test was used. Pearson's $\chi^{2}$ test and Fisher's exact correction were used to assess the relationships between categorical variables among groups. Logistic regression analysis was performed to determine significant predictive factors for intra- and postoperative complications. The Kaplan-Meier and Mantel-Cox tests (log rank test) were used to assess the likelihood of late haemorrhage during the follow-up period. The significance level $\mathrm{p}$ was set to $\leq 0.05$. The study was approved by the Academic Council of the Zarifa Aliyeva National Ophthalmology Centre. Written informed consent was obtained from all patients for the surgery and the study.

\section{RESULTS}

\subsection{Anatomical and Functional Results of Surgery}

In both groups, complete retinal attachment after primary vitrectomy was achieved in all eyes $(100 \%)$. The recurrence of retinal detachment was not observed during the follow-up period (Fig. 1). The functional results are shown in Table 2. In both groups, before surgery, the BCVA was low (1.66 \pm 0.76 and $1.76 \pm 0.80 \operatorname{LogMAR}$, respectively, $\mathrm{p}=0.519)$. Six months after vitrectomy, the BCVA improved in both groups, but the BCVA was significantly higher in the main group than in the control group $(0.66 \pm 0.39$ and $0.89 \pm 0.47$, respectively, $\mathrm{p}=$ $0.008)$. In the main group, the BCVA changed insignificantly at 12 months $(0.67 \pm 0.40)$; however, in the control group, it improved more noticeably (up to $0.75 \pm 0.45$ ) and approached the BCVA of the main group $(\mathrm{p}=0.310)$.

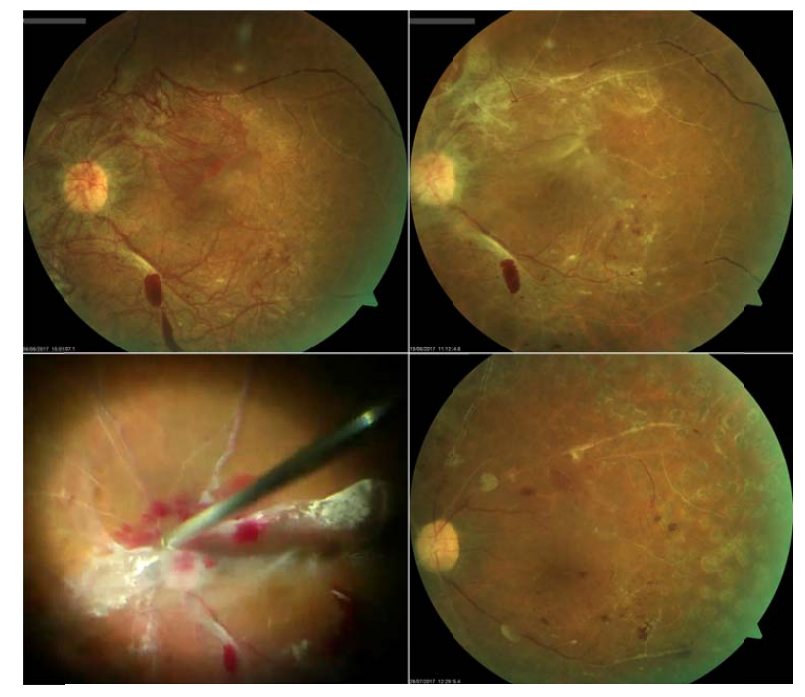

Fig. (1). A 54 years old male patient, had active fibrovascular proliferation with vitreomacular traction in the left eye (top left); 7 days after bevacizumab injection, the retinal neovascularization regressed (top right). During the surgery (vitrectomy with endolaser photocoagulation and gas tamponade), minimal vitreous cavity haemorrhage was observed (lower left), and 1 month after surgery, the retina was attached without early (residual) haemorrhage (lower right).

Table 2. BCVA changes after surgery (in LogMAR units).

\begin{tabular}{|c|c|c|c|}
\hline - & $\begin{array}{c}\text { The Bevacizumab } \\
\text { Group }\end{array}$ & $\begin{array}{c}\text { The Control } \\
\text { Group }\end{array}$ & $\boldsymbol{p}$-value \\
\hline Before surgery & $1.66 \pm 0.76$ & $1.76 \pm 0.80$ & $0,519 \dagger$ \\
\hline At 6 months & $0.66 \pm 0.39$ & $0.89 \pm 0.47$ & $\mathbf{0 , 0 0 8} \dagger$ \\
\hline At 12 months & $0.67 \pm 0.40$ & $0.75 \pm 0.45$ & $0.310 \dagger$ \\
\hline
\end{tabular}

$t-$ Mann-Whitney U test. For $\mathrm{p}<0.05$, the result is highlighted in bold.

\subsection{Intraoperative Complications}

The effect of the preoperative bevacizumab injection on the course of the surgery, the frequency of intraoperative haemorrhage, the use of endodiathermy during the surgery, and the duration of the surgery have been studied (Table $\mathbf{3}$ ). Clinically significant intraoperative haemorrhage was observed more frequently in the control group (in 36 eyes, $51.4 \%$ ) than in the bevacizumab group (in 15 eyes, $31.2 \%$ ), $p=0.030$. The frequency of endodiathermy use was also significantly higher in the control group than in the group with the preoperative bevacizumab injection. Endodiathermy was used in only 9 of 48 eyes $(18.8 \%)$ in the main group and in 27 of 70 eyes $(38.6 \%, p=0.026)$ in the control group. Logistic regression 
analysis showed that the preoperative injection with bevacizumab injection reduced the risk of clinically significant haemorrhage by 2.3 times and the need for endodiathermy by 2.7 times $(p=0.031$ and $p=0.024$, respectively). Although the duration of surgery was shorter in the bevacizumab group than in the control group, the difference was statistically insignificant $(61.70 \pm 18.10$ and $67.13 \pm 19.41$ minutes, respectively, $\mathrm{p}=0.223)$.

\subsection{Postoperative Complications}

The mean follow-up period was $30.00 \pm 20.99$ months in the bevacizumab group and $24.49 \pm 10.22$ months in the control group $(p=0.060)$. We compared the incidence of early and late vitreous cavity haemorrhages and the need for reintervention in both groups after surgery (Table 3 ). To study the frequency of early vitreous cavity haemorrhage, eyes with silicone oil tamponade were excluded from both groups ( 8 eyes from the main group and 12 eyes from the control group). Early vitreous cavity haemorrhage was observed in 6 of 40 eyes $(15.0 \%)$ in the bevacizumab group and in 20 of 58 eyes $(35.5 \%)$ in the control group $(p=0.038)$. Logistic regression analysis showed that the use of the preoperative injection of bevacizumab before vitrectomy reduced the risk of vitreous cavity haemorrhage in the early postoperative period by 3.0 times $(p=0.036)$. At the same time, we did not observe a statistically significant difference in the incidence of late postoperative vitreous cavity haemorrhage between the two groups. Recurrent vitreous cavity haemorrhage during the follow-up period was observed in 8 of 48 eyes $(16.7 \%)$ in the bevacizumab group, and in 12 of 70 eyes $(17.1 \%)$ in the control group $(\mathrm{p}=0.946)$. Recurrent vitreous cavity haemorrhage was observed on average $9.63 \pm 4.14$ months after primary vitrectomy in the group treated with bevacizumab and somewhat earlier, after $6.56 \pm 5.48$ months, in the control group, but the difference was statistically insignificant ( $\mathrm{p}=$ 0.448 ). During the observation period, repeated interventions for recurrent vitreous cavity haemorrhages (washing of the vitreous cavity) were performed in 3 of 48 eyes $(6.2 \%)$ in the group with bevacizumab and in 8 of 70 eyes in the control group $(11.4 \%, \mathrm{p}=0.342)$. In other cases, the preference was given to repeated intravitreal bevacizumab injections or observation.

To assess the risk of late vitreous cavity haemorrhage during the follow-up period, a Kaplan-Meier survival analysis was performed, and no significant differences between the two groups were observed (Fig. 2). A total of $95.8 \%$ and $91.4 \%$ of the eyes under observation in the bevacizumab group and control group, respectively, were free of vitreous cavity haemorrhage 6 months after surgery. These ratios were $84.6 \%$ and $90 \%$ at 12 months and $81.2 \%$ and $79.7 \%$ at 24 months after surgery in the main and control groups, respectively. According to the Mantel-Cox test (logarithmic rank test), the difference was statistically insignificant $\left(\chi^{2}=0.001 ; \mathrm{p}=\right.$ $0.969)$.

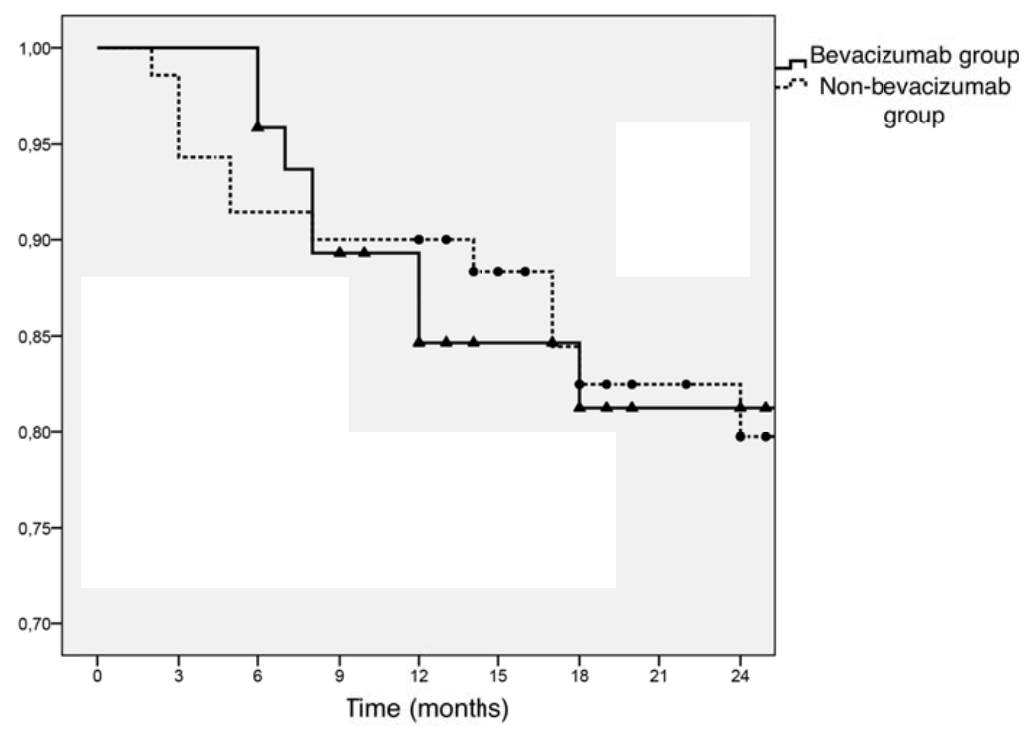

Fig. (2). Risk of recurrent vitreous cavity haemorrhages in different groups (Kaplan-Meier test).

Table 3. The incidence of intra- and postoperative complications in different groups.

\begin{tabular}{|c|c|c|c|c|}
\hline \multicolumn{2}{|l|}{ Variables } & The bevacizumab group & The control group & $p$-value \\
\hline \multicolumn{2}{|l|}{ Number of patients } & 48 & 70 & \\
\hline \multicolumn{2}{|l|}{ Number of eyes } & 48 & 70 & \\
\hline \multirow[t]{2}{*}{ Clinically significant intraoperative haemorrhage } & No & $33(68.8 \%)$ & $34(48.6 \%)$ & \multirow{2}{*}{$0.030 *$} \\
\hline & Yes & $15(31.2 \%)$ & $36(51.4 \%)$ & \\
\hline
\end{tabular}


(Table 3) contd.....

\begin{tabular}{|c|c|c|c|c|}
\hline \multicolumn{2}{|l|}{ Variables } & The bevacizumab group & The control group & p-value \\
\hline \multirow[t]{2}{*}{ Intraoperative endodiathermy use } & No & $39(81.2 \%)$ & $43(61.4 \%)$ & \multirow[t]{2}{*}{$0.022 *$} \\
\hline & Yes & $9(18.8 \%)$ & $27(38.6 \%)$ & \\
\hline \multicolumn{2}{|l|}{ Duration of surgery (in min.) } & $61.70 \pm 18.10$ & $67.13 \pm 19.41$ & $0.223+$ \\
\hline \multicolumn{2}{|l|}{ Follow-up duration (in months) } & $30.00 \pm 20.99(6-74)$ & $24.49 \pm 10.22(12-60)$ & $0.060 \%$ \\
\hline \multirow[t]{2}{*}{ Early postoperative haemorrhage } & No & $34(85.0 \%)$ & $38(64.5 \%)$ & \multirow{2}{*}{$0.038 \%$} \\
\hline & Yes & $6(15.0 \%)$ & $20(35.5 \%)$ & \\
\hline \multirow[t]{2}{*}{ Late postoperative haemorrhage } & No & $40(83.3 \%)$ & $58(82.9 \%)$ & \multirow[t]{2}{*}{0.9467} \\
\hline & Yes & $8(16.7 \%)$ & $12(17.1 \%)$ & \\
\hline \multicolumn{2}{|l|}{ Reoperation (vitreous cavity washout) } & $3(6.2 \%)$ & $8(11.4 \%)$ & $0.342 t$ \\
\hline
\end{tabular}

*- Pearson's $\chi^{2}$ test, $\uparrow-$ Mann-Whitney U test, $\not-$ Fisher exact test. For $\mathrm{p}<0.05$, the result is highlighted in bold.

\section{DISCUSSION}

Vascular Endothelial Growth Factor (VEGF) is a regulator of angiogenesis and vascular permeability and plays a key role in the pathogenesis of diabetic retinopathy and other vascular diseases of the retina $[9,21]$. Vascular endothelial growth factor inhibitors (anti-VEGF agents) have been successfully used in ophthalmology for the regression of neovascularization and reduction of macular oedema, particularly in the treatment of intraocular haemorrhages, neovascular glaucoma and macular oedema in PDR $[10,22]$. The most popular anti-VEGF agents currently used in ophthalmology are ranibizumab (Lucentis, Genentech, USA), bevacizumab (Avastin, Genentech, USA) and aflibercept (Eylea, Regeneron, USA).

Bevacizumab is a humanized monoclonal antibody that binds all VEGF-A isoforms. Chen E. and Park C.H. for the first time in 2006, reported the use of bevacizumab as an adjunct before vitrectomy and suggested that the inhibition of new vessels may reduce the risk of intraoperative haemorrhage and facilitate the operation [11]. Later, several studies reported that the preoperative administration of bevacizumab positively affects vitrectomy and the incidence of complications [12 - 16, 23 - 27]. The authors noted that the use of bevacizumab facilitated fibrovascular membrane dissection, segmentation and delamination and improved visualization during surgery. Other advantages were less frequent use of endodiathermy and shortening of surgery time $[13,26]$. These authors have also reported a decrease in the incidence of vitreous cavity haemorrhage, especially early haemorrhage, after vitrectomy in patients who received a preoperative injection of bevacizumab. Other studies in the literature have similarly reported positive effects of the preoperative use of other anti-VEGF agents, such as ranibizumab [28], aflibercept [29], and conbercept [30].

Nevertheless, the usefulness of anti-VEGF agents as adjuvants before vitrectomy have been questioned in several studies [17 - 20]. Lo et al. compared the results of vitrectomy in 33 eyes that received a preoperative bevacizumab injection and 104 eyes that did not receive the injection and concluded that the preoperative injection of bevacizumab does not affect the incidence of postoperative haemorrhage or the final visual acuity [17]. Ahn et al. performed a randomized study of vitrectomy in which bevacizumab was used before surgery or during surgery or not used, and the authors did not find a significant difference in the incidence of postoperative complications [18]. Farahvash et al. studied the effect of the preoperative use of bevacizumab in dense vitreous haemorrhages and concluded that the use of anti-VEGF agents did not have a positive effect on the course of surgery or the incidence of early postoperative complications [19].

Our results confirm the benefits of bevacizumab as an adjuvant to vitrectomy in eyes with complications of PDR in general. The incidence of intraoperative haemorrhage in the eyes that received the bevacizumab injection was $31.2 \%$, and in the control group, which did not receive preoperative injections, it was $51.4 \%(\mathrm{p}=0.030)$. The endodiathermy was also used less frequently in the main group than in the control group $(18.8 \%$ and $38.6 \%$, respectively, $\mathrm{p}=0.022)$. In addition, logistic regression analysis showed that the use of preoperative injections of bevacizumab reduced the risk of intraoperative haemorrhage by 2.3 times and the need for endodiathermy by 2.7 times $(p=0.031$ and $p=0.024$, respectively). However, we did not find a significant difference in the duration of surgery between the two groups.

Intravitreal injections of bevacizumab before surgery also reduced the risk of early postoperative vitreous cavity haemorrhage by 3.0 times $(p=0.036)$ but did not significantly affect the incidence of late vitreous cavity haemorrhage. The incidence of late postoperative vitreous cavity haemorrhage was $16.7 \%$, in the bevacizumab group and $17.1 \%$ in the control group. In the bevacizumab group, the average visual acuity at 6 months after surgery was significantly higher than that in the control group $(0.66 \pm 0.39$ and $0.89 \pm 0.47$ in LogMAR units, respectively, $\mathrm{p}=0.008)$, but at 12 months, visual acuity was similar in both groups $(0.67 \pm 0.40$ and $0.75 \pm 0.45$, respectively, $\mathrm{p}=0.310$ ).

\section{CONCLUSION}

Our observations show that the preoperative use of bevacizumab as an adjunct to diabetic vitrectomy can help to reduce the incidence of intraoperative and early postoperative vitreous cavity haemorrhage, which leads to better functional results in the early postoperative period. Over the long-term follow-up period, the effect of the preoperative bevacizumab injections is declining, which is reflected in the absence of a difference in the frequency of late vitreous cavity haemorrhages and the functional outcomes of the surgery.

\section{ETHICS APPROVAL AND CONSENT TO PARTICIPATE}

The study design was approved by the ethics committee of the National Ophthalmology Centre named acad. Zarifa Aliyeva, Baku, Azerbaijan (Reference number 341). 


\section{HUMAN AND ANIMAL RIGHTS}

No Animals were used in this research. All human research procedures followed were in accordance with the ethical standards of the committee responsible for human experimentation (institutional and national), and with the Helsinki Declaration of 1975, as revised in 2013.

\section{CONSENT FOR PUBLICATION}

Informed consent was obtained from all the participants.

\section{AVAILABILITY OF DATA AND MATERIALS}

The data and materials used to support the findings of this study are available from the corresponding author [L.A] upon request.

\section{FUNDING}

None.

\section{CONFLICT OF INTEREST}

The authors declare no conflict of interest, financial or otherwise

\section{ACKNOWLEDGEMENTS}

Declared none.

\section{REFERENCES}

[1] Zheng Y, He M, Congdon N. The worldwide epidemic of diabetic retinopathy. Indian J Ophthalmol 2012; 60(5): 428-31. [http://dx.doi.org/10.4103/0301-4738.100542] [PMID: 22944754]

[2] Schachat AP, Oyakawa RT, Michels RG, Rice TA. Complications of vitreous surgery for diabetic retinopathy. II. Postoperative complications. Ophthalmology 1983; 90(5): 522-30. [http://dx.doi.org/10.1016/S0161-6420(83)34540-1] [PMID: 6192378]

[3] Novak MA, Rice TA, Michels RG, Auer C. Vitreous hemorrhage after vitrectomy for diabetic retinopathy. Ophthalmology 1984; 91(12): 1485-9.

[http://dx.doi.org/10.1016/S0161-6420(84)34099-4] [PMID: 6521989]

[4] Blankenship GW. Management of vitreous cavity hemorrhage following pars plana vitrectomy for diabetic retinopathy. Ophthalmology 1986; 93(1): 39-44

[http://dx.doi.org/10.1016/S0161-6420(86)33791-6] [PMID: 2419815]

[5] Tolentino FI, Cajita VN, Gancayco T, Skates S. Vitreous hemorrhage after closed vitrectomy for proliferative diabetic retinopathy. Ophthalmology 1989; 96(10): 1495-500.

[http://dx.doi.org/10.1016/S0161-6420(89)32700-X] [PMID: 2587044]

[6] Neely KA, Scroggs MW, McCuen BW II. Peripheral retinal cryotherapy for postvitrectomy diabetic vitreous hemorrhage in phakic eyes. Am J Ophthalmol 1998; 126(1): 82-90

[http://dx.doi.org/10.1016/S0002-9394(98)00066-X] [PMID: 9683153]

[7] Yeh PT, Yang CM, Yang CH, Huang JS. Cryotherapy of the anterior retina and sclerotomy sites in diabetic vitrectomy to prevent recurrent vitreous hemorrhage: an ultrasound biomicroscopy study. Ophthalmology 2005; 112(12): 2095-102. [http://dx.doi.org/10.1016/j.ophtha.2005.07.010] [PMID: 16225926]

[8] Yang CM, Yeh PT, Yang CH. Intravitreal long-acting gas in the prevention of early postoperative vitreous hemorrhage in diabetic vitrectomy. Ophthalmology 2007; 114(4): 710-5. [http://dx.doi.org/10.1016/j.ophtha.2006.07.047] [PMID: 17275908]

[9] Wang DY, Zhao XY, Zhang WF, Meng LH, Chen YX. Perioperative anti-vascular endothelial growth factor agents treatment in patients undergoing vitrectomy for complicated proliferative diabetic retinopathy: a network meta-analysis. Sci Rep 2020; 10(1): 18880. [http://dx.doi.org/10.1038/s41598-020-75896-8]

[10] Osaadon P, Fagan XJ, Lifshitz T, Levy J. A review of anti-VEGF agents for proliferative diabetic retinopathy. Eye (Lond) 2014; 28(5): 510-20.

[http://dx.doi.org/10.1038/eye.2014.13] [PMID: 24525867]

[11] Chen E, Park CH. Use of intravitreal bevacizumab as a preoperative adjunct for tractional retinal detachment repair in severe proliferative diabetic retinopathy. Retina 2006; 26(6): 699-700.

[http://dx.doi.org/10.1097/01.iae.0000225351.87205.69] [PMID: 16829817]

[12] Yeoh J, Williams C, Allen P, et al. Avastin as an adjunct to vitrectomy in the management of severe proliferative diabetic retinopathy: a prospective case series. Clin Exp Ophthalmol 2008; 36(5): 449-54. [http://dx.doi.org/10.1111/j.1442-9071.2008.01805.x] [PMID: 18942219]

[13] Rizzo S, Genovesi-Ebert F, Di Bartolo E, Vento A, Miniaci S, Williams G. Injection of intravitreal bevacizumab (Avastin) as a preoperative adjunct before vitrectomy surgery in the treatment of severe proliferative diabetic retinopathy (PDR). Graefes Arch Clin Exp Ophthalmol 2008; 246(6): 837-42.

[http://dx.doi.org/10.1007/s00417-008-0774-y] [PMID: 18286296]

[14] El-Batarny AM. Intravitreal bevacizumab as an adjunctive therapy before diabetic vitrectomy. Clin Ophthalmol 2008; 2(4): 709-16. [PMID: 19668420]

[15] Ahmadieh H, Shoeibi N, Entezari M, Monshizadeh R. Intravitrea bevacizumab for prevention of early postvitrectomy hemorrhage in diabetic patients: a randomized clinical trial. Ophthalmology 2009; 116(10): 1943-8

[http://dx.doi.org/10.1016/j.ophtha.2009.07.001] [PMID: 19699531]

[16] Yeh PT, Yang CM, Lin YC, Chen MS, Yang CH. Bevacizumab pretreatment in vitrectomy with silicone oil for severe diabetic retinopathy. Retina 2009; 29(6): 768-74.

[http://dx.doi.org/10.1097/IAE.0b013e3181a3b7ef] [PMID: 19516117]

[17] Lo WR, Kim SJ, Aaberg TM Sr, et al. Visual outcomes and incidence of recurrent vitreous hemorrhage after vitrectomy in diabetic eyes pretreated with bevacizumab (avastin). Retina 2009; 29(7): 926-31. [http://dx.doi.org/10.1097/IAE.0b013e3181a8eb88] [PMID: 19584650]

[18] Ahn J, Woo SJ, Chung H, Park KH. The effect of adjunctive intravitreal bevacizumab for preventing postvitrectomy hemorrhage in proliferative diabetic retinopathy. Ophthalmology 2011; 118(11): 2218-26.

[http://dx.doi.org/10.1016/j.ophtha.2011.03.036] [PMID: 21724263]

[19] Farahvash MS, Majidi AR, Roohipoor R, Ghassemi F. Preoperative injection of intravitreal bevacizumab in dense diabetic vitreous hemorrhage. Retina 2011; 31(7): 1254-60.

[http://dx.doi.org/10.1097/IAE.0b013e31820a68e5] [PMID: 21499192]

[20] Sato T, Morita S, Bando H, Sato S, Ikeda T, Emi K. Early vitreous hemorrhage after vitrectomy with preoperative intravitreal bevacizumab for proliferative diabetic retinopathy. Middle East Afr J Ophthalmol 2013; 20(1): 51-5.

[http://dx.doi.org/10.4103/0974-9233.106387] [PMID: 23580852]

[21] Ferrara N. Role of vascular endothelial growth factor in physiologic and pathologic angiogenesis: therapeutic implications. Semin Oncol 2002; 29(6)(Suppl. 16): 10-4

[http://dx.doi.org/10.1016/S0093-7754(02)70064-X] 12516033]

[22] Salam A, Mathew R, Sivaprasad S. Treatment of proliferative diabetic retinopathy with anti-VEGF agents. Acta Ophthalmol 2011; 89(5): 405-11.

[http://dx.doi.org/10.1111/j.1755-3768.2010.02079.x]

[PMID: 21294854]

[23] Modarres M, Nazari H, Falavarjani KG, Naseripour M, Hashemi M, Parvaresh MM. Intravitreal injection of bevacizumab before vitrectomy for proliferative diabetic retinopathy. Eur J Ophthalmol 2009; 19(5): 848-52.

[http://dx.doi.org/10.1177/112067210901900526] [PMID: 19787608]

[24] Romano MR, Gibran SK, Marticorena J, Wong D, Heimann H. Can a preoperative bevacizumab injection prevent recurrent postvitrectomy diabetic vitreous haemorrhage? Eye (Lond) 2009; 23(8): 1698-701.

[http://dx.doi.org/10.1038/eye.2008.354] [PMID: 19039332]

[25] Yeung L, Liu L, Wu WC, et al. Reducing the incidence of early postoperative vitreous haemorrhage by preoperative intravitreal bevacizumab in vitrectomy for diabetic tractional retinal detachment. Acta Ophthalmol 2010; 88(6): 635-40.

[http://dx.doi.org/10.1111/j.1755-3768.2008.01498.x]

[PMID: 19432872]

[26] di Lauro R, De Ruggiero P, di Lauro R, di Lauro MT, Romano MR. 
Intravitreal bevacizumab for surgical treatment of severe proliferative diabetic retinopathy. Graefes Arch Clin Exp Ophthalmol 2010; 248(6): 785-91.

[http://dx.doi.org/10.1007/s00417-010-1303-3] [PMID: 20135139]

[27] Gupta A, Bansal R, Gupta V, Dogra MR. Six-month visual outcome after pars plana vitrectomy in proliferative diabetic retinopathy with or without a single preoperative injection of intravitreal bevacizumab. Int Ophthalmol 2012; 32(2): 135-44.

[http://dx.doi.org/10.1007/s10792-012-9541-5] [PMID: 22450558]

[28] Hu L, Chen Q, Du Z, Wang W, Zhao G. Evaluation of vitrectomy combined preoperative intravitreal ranibizumab and postoperative intravitreal triamcinolone acetonide for proliferative diabetic retinopathy. Int Ophthalmol 2021; 41(5): 1635-42. [published online ahead of print, $2021 \mathrm{Feb} 4]$

[http://dx.doi.org/10.1007/s10792-021-01703-6] [PMID: 33538931]

[29] Umanets N, Korol A, Vit V, Zavodnaya V, Pasyechnikova N. Peculiarities of vitrectomy and morphologic changes in the epiretinal membrane after intravitreal aflibercept in patients with severe proliferative diabetic retinopathy. Retin Cases Brief Rep 2017; 11(2): $114-8$.

[http://dx.doi.org/10.1097/ICB.0000000000000306]

[PMID: 27100568

[30] Su L, Ren X, Wei H, et al. Intravitreal conbercept (KH902) for surgical treatment of severe proliferative diabetic retinopathy. Retina 2016; 36(5): 938-43

[http://dx.doi.org/10.1097/IAE.0000000000000900] [PMID: 26630313]

\section{(C) 2021 Karimov and Akhundova.}

This is an open access article distributed under the terms of the Creative Commons Attribution 4.0 International Public License (CC-BY 4.0), a copy of which is available at: https://creativecommons.org/licenses/by/4.0/legalcode. This license permits unrestricted use, distribution, and reproduction in any medium, provided the original author and source are credited. 